\title{
Differential Localization of Glutamate Receptor Subunits at the Drosophila Neuromuscular Junction
}

\author{
Scott B. Marrus, ${ }^{1}$ Scott L. Portman, ${ }^{1}$ Marcus J. Allen, ${ }^{2}$ Kevin G. Moffat, ${ }^{2}$ and Aaron DiAntonio ${ }^{1}$ \\ ${ }^{1}$ Department of Molecular Biology and Pharmacology, Washington University School of Medicine, St. Louis, Missouri 63110, and ${ }^{2}$ Department of Biological \\ Sciences, University of Warwick, Coventry CV4 7AL, United Kingdom
}

The subunit composition of postsynaptic neurotransmitter receptors is a key determinant of synaptic physiology. Two glutamate receptor subunits, Drosophila glutamate receptor IIA (DGluRIIA) and DGluRIIB, are expressed at the Drosophila neuromuscular junction and are redundant for viability, yet differ in their physiological properties. We now identify a third glutamate receptor subunit at the Drosophila neuromuscular junction, DGluRIII, which is essential for viability. DGluRIII is required for the synaptic localization of DGluRIIA and DGluRIIB and for synaptic transmission. Either DGluRIIA or DGluRIIB, but not both, is required for the synaptic localization of DGluRIII. DGluRIIA and DGluRIIB compete with each other for access to DGluRIII and subsequent localization to the synapse. These results are consistent with a model of a multimeric receptor in which DGluRIII is an essential component. At single postsynaptic cells that receive innervation from multiple motoneurons, DGluRIII is abundant at all synapses. However, DGluRIIA and DGluRIIB are differentially localized at the postsynaptic density opposite distinct motoneurons. Hence, innervating motoneurons may regulate the subunit composition of their receptor fields within a shared postsynaptic cell. The capacity of presynaptic inputs to shape the subunit composition of postsynaptic receptors could be an important mechanism for synapse-specific regulation of synaptic function and plasticity.

Key words: glutamate receptor; Drosophila; synapse; neuromuscular junction; synaptic transmission; afferent

\section{Introduction}

Changes in synaptic strength are important for the development of neuronal circuits and their modification by experience. The postsynaptic response to transmitter is a key determinant of synaptic strength and is a substrate on which plasticity mechanisms can act. The central mechanism that controls the response to transmitter is the function of the postsynaptic neurotransmitter receptors. Subunit composition and synaptic localization are two key mechanisms that regulate the function of these receptors.

The role of subunit composition in the assembly and trafficking of vertebrate AMPA-type glutamate receptors has been studied extensively (Barry and Ziff, 2002; Malinow and Malenka, 2002; McGee and Bredt, 2003). The various receptor subunits differ in a number of physiological parameters, including desensitization kinetics and ion permeability (Hollmann and Heinemann, 1994; Westbrook, 1994). The differential utilization of

Received June 24, 2003; revised Dec. 19, 2003; accepted Dec. 19, 2003.

This work was supported by National Institutes of Health Grant R01 NS043171 to A.D. as well as the Keck, McKnight, Howard Hughes Medical Institute, Burroughs Wellcome, and Sloan Foundations. S.B.M. was supported by a National Research Service Award Training Grant NS44754-01. We thank Jason Lee and Angela Wilkins for excellent technical assistance and Dr. Corey Goodman, in whose laboratory some initial experiments were performed. We thank Natasha Viquez for critical reading of this manuscript.

Correspondence should be addressed to Aaron DiAntonio, Department of Molecular Biology and Pharmacology, Washington University School of Medicine, Campus Box 8103, 660 South Euclid, St. Louis, M0 63110. E-mail: diantonio@wustl.edu.

M. J. Allen's present address: Research School of Biosciences, University of Kent at Canterbury, Canterbury, Kent CT2 7NJ, UK.

DOI:10.1523/JNEUROSCI.1575-03.2004

Copyright $\odot 2004$ Society for Neuroscience $\quad$ 0270-6474/04/241406-10\$15.00/0 these subunits alters the properties of the resulting postsynaptic receptors (Jonas et al., 1994; Washburn et al., 1997). Subunit composition also regulates the assembly, insertion, synaptic localization, and anchoring of AMPA receptors (Barry and Ziff, 2002; Malinow and Malenka, 2002; McGee and Bredt, 2003). One mechanism that may influence the subunit composition of the postsynaptic transmitter receptor is the identity of the presynaptic afferent (Toth and McBain, 1998, 2000; Craig and Boudin, 2001). Physiological studies of single hippocampal neurons demonstrate that the AMPA receptors opposite mossy fiber and area CA3 inputs are pharmacologically distinct (Toth and McBain, 1998).

The Drosophila neuromuscular junction (NMJ) is a glutamatergic synapse that expresses postsynaptic receptors that are homologous to vertebrate AMPA and kainate receptors. Two muscle receptors have been identified, Drosophila glutamate receptor IIA (DGluRIIA) and DGluRIIB, that differ in their physiological properties (Schuster et al., 1991; Petersen et al., 1997; Davis et al., 1998; DiAntonio et al., 1999). Receptors that differ in their subunit composition display differences in their single-channel behavior that affects their synaptic response (DiAntonio et al., 1999). Because DGluRIIA and DGluRIIB are redundant for viability, neither must be essential for assembly or localization of functional glutamate receptors. However, it is unknown what role these receptors may play in glutamate receptor localization or what other molecules and mechanisms regulate the localization of functional glutamate receptors to the Drosophila NMJ.

Here, we report the identification and characterization of a 
novel glutamate receptor subunit that is present at the Drosophila NMJ, which we named DGluRIII. We find that DGluRIII is essential for viability and is necessary for the synaptic localization of DGluRIIA and DGluRIIB and for synaptic transmission. In contrast, either DGluRIIA or DGluRIIB, but not both, are required for the synaptic localization of DGluRIII. DGluRIIA and DGluRIIB compete with each other for access to DGluRIII and subsequent localization to the synapse. At postsynaptic cells that are multi-innervated, DGluRIIA and DGluRIIB are differentially localized to the NMJs of distinct motoneurons. These studies define the logic of subunit assembly at the Drosophila neuromuscular junction and suggest that the presynaptic input shapes the subunit composition of its receptor field.

\section{Materials and Methods \\ Genetics}

DGluRIII mutagenesis. Ethyl methysulfonate was used to mutagenize an isogenized white Canton S stock. An F2 lethal screen was performed over the deficiency $\mathrm{df}(2 \mathrm{~L})$ ast 4 that covers the 21D1-2;21E1-2 region and identified 55 mutant alleles. One complementation group containing two alleles represents the DGluRIII gene, because it can be rescued by a DGluRIII genomic transgene. These alleles act as genetic nulls because the homozygous phenotype is the same as the phenotype of the mutant over a deficiency. The mutants in DGluRIIA and DGluRIIB used in this study have been described previously (Petersen et al., 1997; DiAntonio et al., 1999). In brief, DGluRIIA null $=$ DGluRIIA ${ }^{A D 9} / D G l u R I I A \& B^{S P 22}$ (DGluRIIA null in combination with a DGluRIIA and DGluRIIB double mutant); DGluRIIB null = DGluRIIA\& $B^{S P 22} / d f(2 L) c l^{h 4} ; P[g D G l u R I I A] /$ + (DGluRIIA and DGluRIIB double mutant in combination with a deficiency that removes both DGluRIIA and DGluRIIB rescued with a genomic DGluRIIA rescue construct). We demonstrated previously that this genomic rescue transgene of DGluRIIA functions in a manner that is quantitatively very similar to the endogenous DGluRIIA gene [Petersen et al., (1997), their Fig. 4]. For the DGluRIIA hypomorph (DGluRIIB null shown in Figure $6 d)$, the full genotype is DGluRIIA\& $B^{S P 22} / d f(2 L) c l^{h 4}$; H94Gal4/P[UAS-DGluRIIAmyc]. This is a DGluRIIA and DGluRIIB double mutant rescued by the low-level expression of a DGluRIIA transgene (DiAntonio et al., 1999).

Transgenic constructs. The DGluRIII cDNA was cloned into the pUAST vector (Brand and Perrimon, 1993) and into a transformation vector containing the myosin heavy chain (MHC) promoter (Petersen et al., 1997). Genomic rescue constructs were generated by PCR using a $5^{\prime}$ oligo GCTCTCAGCTACACATGTCG and a 3' oligo GACGTAGCTGTAGAGCACAAC, and the product was sequenced and cloned into the pUAST vector. Transformants were generated by standard techniques.

\section{Immunohistochemistry}

Antibodies. The mouse monoclonal anti-DGluRIIA antibody 8B4D2, developed by Christoph Schuster (Max Planck Gesellschaft, Tubingen, Germany) and Corey Goodman (Renovis, San Francisco, CA), was obtained from the Developmental Studies Hybridoma Bank (University of Iowa, Iowa City, IA), developed under the auspices of the National Institute of Child Health and Human Development, and maintained by the University of Iowa. It was used at a 1:100 dilution on larvae fixed for 5 min with Bouin's fixative. The rabbit anti-DGluRIIB antibody was raised by Sigma-Genosys (St. Louis, MO) against the $15 \mathrm{C}$-terminal residues of the predicted DGluRIIB gene product (-ASSAKKKKKTRRIEK), affinity purified and used at 1:2500 dilution. There is no detectable staining in flies that are genetically null for $D G l u R I I B$, but staining appears normal in flies null for DGluRIIA. For both DGluRIIA and DGluRIIB, we occasionally see extra synaptic puncta. These are not consistently observed and, when present, do not show any colocalization between the two receptor subunits. The rabbit anti-DGluRIII antibody was raised by Zymed (San Francisco, CA) against the 22 C-terminal residues of the predicted DGluRIII gene product (-QGSGSSSGSNNAGRGEKEARV), affinity purified, and used at 1:2500 dilution. Synaptic staining appears normal in flies lacking either DGluRIIA or DGluRIIB. Neither the antisera to DGluRIIB nor DGluRIII work on immunoblots. The mouse monoclonal anti-fasciclin II (FasII) (1D4) antibody, developed by Corey Goodman, was obtained from Developmental Studies Hybridoma Bank and used at 1:5 dilution. The Cy3 (indocarbocyanine)-conjugated goat anti-HRP antibody was obtained from Jackson ImmunoResearch (West Grove, PA) and used at 1:2000 dilution. Secondary antibodies were obtained from Molecular Probes (Eugene, OR) and used at 1:1000 dilution.

Anatomy. Embryonic RNA in situs were performed as described previously (Tautz and Pfeifle, 1989). Immunohistochemistry was performed on wandering third-instar larvae. Larvae were pinned on Sylgard-lined Petri dishes with insect pins. After dissecting open the dorsal side of the larvae and removing the internal organs and trachea, the larvae were pinned flat for fixation. Larvae were fixed for $5 \mathrm{~min}$ in either $3.7 \%$ formaldehyde in PBS or in Bouin's fixative (necessary for anti-DGluRIIA staining). Larvae were incubated with the primary antibodies described above, followed by the appropriate secondary antibodies, equilibrated in $70 \%$ glycerol, and mounted in VectaShield (Vector Laboratories, Burlingame, CA) mounting media.

Imaging and analysis. Larvae were imaged on a Nikon (Tokyo, Japan) $\mathrm{C} 1$ confocal microscope. All images of mutant larvae were acquired in conjunction with a wild-type control that had been simultaneously stained. The same confocal gain setting was used for both the wild type and mutant larvae. The gain was chosen as the maximum gain that did not saturate the signal from wild-type larvae. A complete Z-stack was acquired for each NMJ and rendered as a maximum projection. Quantitative analysis of fluorescence intensity was done using MetaMorph software (Universal Imaging Corporation, West Chester, PA). The differential localization of DGluRIIA and DGluRIIB demonstrated in Figure $8 b$ does not depend on the color of the secondary antibody used.

\section{Electrophysiology}

Current clamp. Intracellular recordings were performed from muscle 6 , segments A3 and A4, of third-instar female larvae. The larvae were visualized on a Nikon compound microscope with a fixed stage and differential interference contrast optics. Sharp electrodes (15-20 M $\Omega$ ), made of borosilicate glass (outer diameter, $1.2 \mathrm{~mm}$ ), were filled with $3 \mathrm{M} \mathrm{KCl}$. The signal was acquired with an Axoclamp 2B amplifier (Axon Instruments, Foster City, CA), further amplified and filtered at $1 \mathrm{kHz}$ with a Brownlee (San Jose, CA) Precision amplifier (Model 410), digitized with a Digidata 1320A analog-to-digital board, and stored on a personal computer using pClamp 9.0 (Axon Instruments). Input resistance was determined by injecting $1 \mathrm{nA}$ into the muscle for $500 \mathrm{msec}$. Only cells with an input resistance of $\geq 5 \mathrm{M} \Omega$ and a resting potential between -60 and -80 $\mathrm{mV}$ were included in the analysis. Recordings were performed in HL3 (hemolymph-like 3) Stewart saline (Stewart et al., 1994) that contained (in mM): $70 \mathrm{NaCl}, 5 \mathrm{KCl}, 0.45 \mathrm{CaCl}_{2}, 20 \mathrm{MgCl}_{2}, 10 \mathrm{HCO}_{3}, 5$ trehalose, 115 sucrose, and 5 HEPES, $\mathrm{pH}$ adjusted to 7.2. There were only minor differences in the resting membrane potential and the input resistance between wild-type and mutant larvae. Average values were as follows. $\left(V_{m}=\right.$ $\left.\mathrm{mV} \pm \mathrm{SE} ; R_{\mathrm{in}}=\mathrm{M} \Omega \pm \mathrm{SE}\right):(1) w-/$ Canton $S(n=10) V_{m}=70.9 \pm 1.7$; $R_{\text {in }}=8.3 \pm 0.7$, (2) DGluRIII $/ d f(2 L)$ ast $1 ; P[$ UAS-cDGluRIII $] /+(n=$ 11) $V_{m}=68 \pm 1.7 ; R_{\text {in }}=5.7 \pm 0.4$. For analysis of excitatory junctional current kinetics, responses from type Ib and type Is synapses were isolated following the method of Lnenicka and Keshishian (2000).

\section{Results \\ DGluRIII encodes a novel glutamate receptor that is expressed in the somatic musculature}

To date, six ionotropic glutamate receptors have been described in Drosophila. Four of these are expressed exclusively in the CNS (Ultsch et al., 1992, 1993; Vèolkner et al., 2000), whereas two receptors, DGluRIIA and DGluRIIB, are expressed in somatic muscle (Schuster et al., 1991; Petersen et al., 1997). The muscle receptors DGluRIIA and DGluRIIB localize to the neuromuscular junction and form part of the functional receptor complex (Petersen et al., 1997; DiAntonio et al., 1999). However, neither of these receptors is essential and, on the basis of the multimeric nature of vertebrate glutamate receptors (Dingledine et al., 1999; Barry and Ziff, 2002), we reasoned that other subunits might 
exist. We searched for candidate molecules from among the many Drosophila genes that are predicted to encode glutamate receptors (Littleton and Ganetzky, 2000). We focused on one predicted gene, CG4226, because it shows the greatest sequence identity to the muscle receptors DGluRIIA and DGluRIIB (Fig. 1). Embryonic whole-mount in situ hybridization demonstrates that CG4226 RNA is expressed in the somatic musculature but is not detected in the CNS of late-stage embryos (Fig. 2a) and thirdinstar larvae (data not shown). We named this receptor DGluRIII.

DGluRIII exhibits 35-39\% amino acid identity to the previously described DGluRIIA and DGluRIIB, respectively (Fig. 1a) (Schuster et al., 1991; Petersen et al., 1997), and 22\% identity to the neuronal DGluRI (Ultsch et al., 1992). Figure $1 b$ diagrams the predicted evolutionary relationship between Drosophila and mammalian glutamate receptors. Although DGluRIII is a member of the AMPA-kainate superfamily, it cannot be classified as AMPA- or kainate-type on the basis of sequence. Vertebrate AMPA receptors are localized to synapses via interactions with PSD-95/Dlg/ZO-1 (PDZ)-domain proteins (Sheng and Pak, 1999). Neither of the previously characterized Drosophila muscle receptors contain recognizable PDZ-binding motifs; however, DGluRIII exhibits a class II C-terminal consensus PDZ-binding domain site [-EARV; -X- $\Phi-\mathrm{X}-\Phi$, where $\Phi$ is any hydrophobic amino acid (Hung and Sheng, 2002)]. Based on the sequence and expression data for DGluRIII, we chose to undertake a genetic, physiological, and cell biological analysis of its function.

\section{DGluRIII is localized opposite active zones at the NMJ}

We studied the distribution and composition of the native glutamate receptor, so antibodies were required for all three glutamate receptor subunits. A monoclonal antibody to DGluRIIA (8B4D2) was previously reported; however, its specificity for DGluRIIA had not been tested. We found that this antibody robustly stains the NMJ of both wild-type and DGluRIIB null mutant larvae. However, staining is undetectable in larvae that are genetic nulls for DGluRIIA (Fig. $3 a-c$ ). Hence, this monoclonal is specific for DGluRIIA. We raised antibodies to peptide antigens from both DGluRIIB and DGluRIII. The peptides were selected from regions that do not share homology among the three receptors or with any other predicted Drosophila proteins. The antiDGluRIIB antisera stains the NMJ of both wild-type and DGluRIIA null larvae but does not stain larvae that are genetic nulls for DGluRIIB (Fig. $3 d-f$ ). Hence, this sera is specific for the DGluRIIB subunit. Because DGluRIII is reported here for the first time, we describe the staining pattern of the anti-DGluRIII sera in detail below.

DGluRIII protein is localized to the neuromuscular junction at every muscle (Fig. 2b) (data not shown). Transmitter release occurs from presynaptic specializations termed active zones. We previously demonstrated that epitope-tagged transgenic glutamate receptors are concentrated in puncta that lie opposite the presynaptic active zones, placing them in an ideal position to detect the release of glutamate (Petersen et al., 1997). We investigated whether endogenous DGluRIII has the same distribution. The absence of staining for the periactive zone marker FasII defines active-zone regions at synaptic boutons (Fig. $2 d$ ) (Sone et al., 2000; Wan et al., 2000). We found that DGluRIII localizes to puncta that precisely fill the holes in the FasII stain (Fig. $2 c-e$ ), demonstrating its presence opposite active zones. In addition, we previously used immunoelectron microscopy to demonstrate that the myc-tagged DGluRIIA is present opposite each active zone (genotype, MHC-DGluRIIAmyc) (Petersen et al., 1997). a

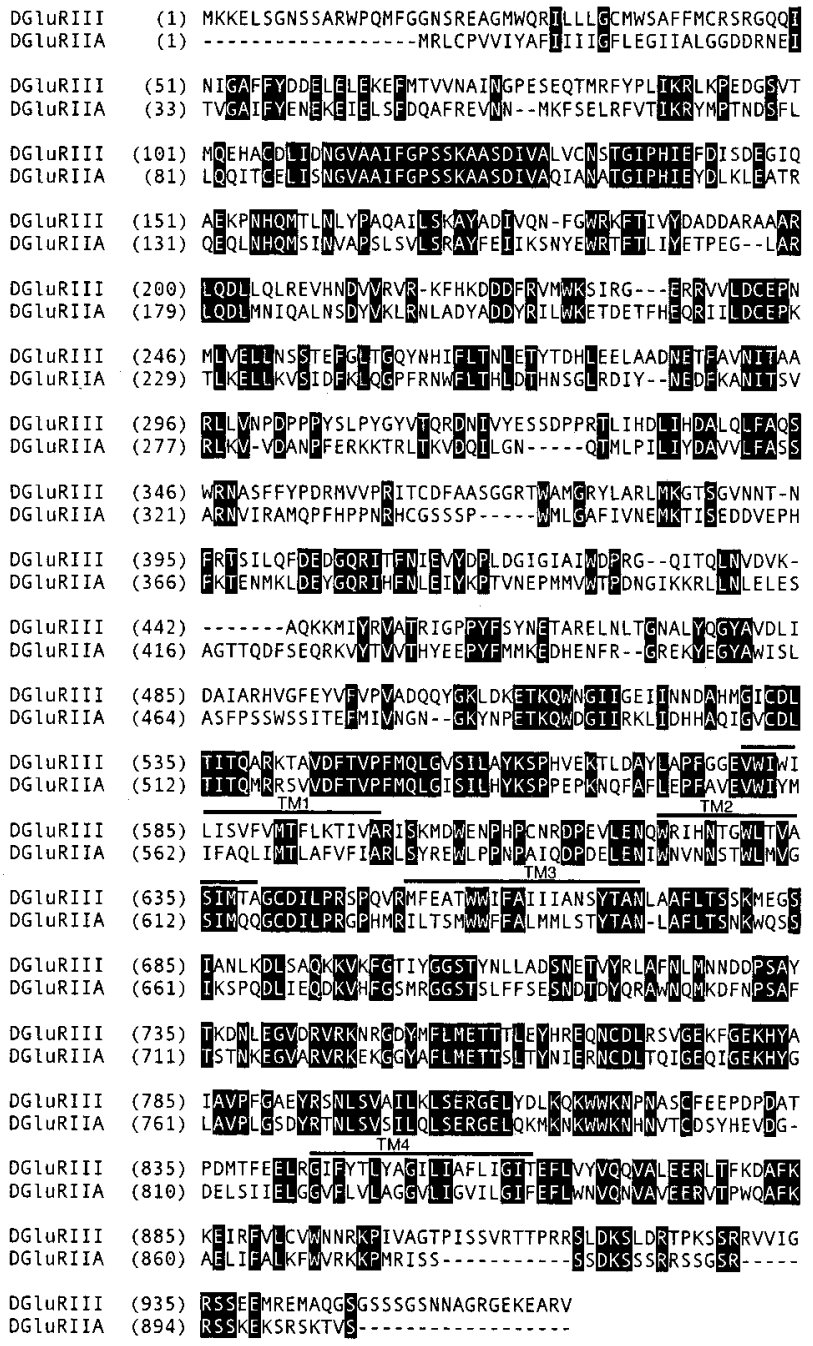

b

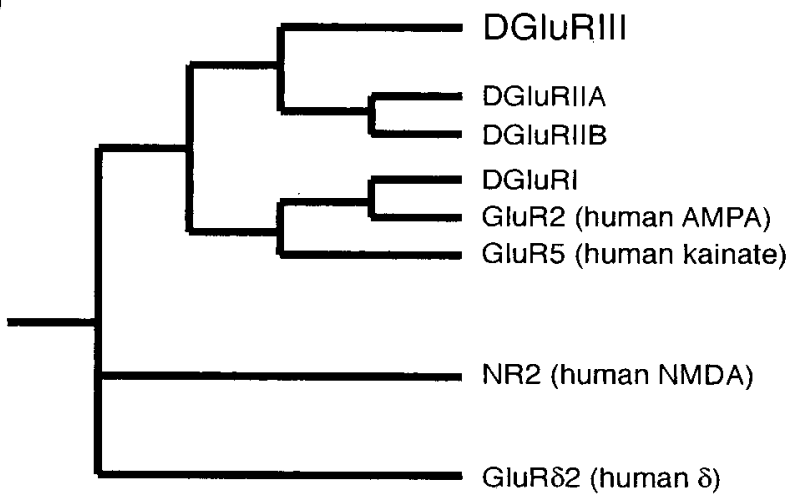

Figure 1. Sequence of DGluRIII. $a$, The predicted amino acid sequences of DGluRIII and DGluRIIA are aligned, and identical amino acids are shaded. The putative transmembrane domains (TM1-TM4) are indicated. $b$, Dendrogram illustrating the predicted evolutionary relationships among glutamate receptors. Dendrogram was generated with the AlignX software in the VectorNTI package.

DGluRIII colocalizes with this tagged DGluRIIA and appears to be present at every punctum (Fig. $2 f-h$ ). This confirms that DGluRIII localizes opposite active zones and suggests that it is a component of the receptor complex at each release site. 


\section{DGluRIII is an essential gene}

To investigate the role of each subunit in the assembly and localization of glutamate receptors, we needed mutations in each gene. We previously generated mutations in DGluRIIA and DGluRIIB (Petersen et al., 1997; DiAntonio et al., 1999). To identify a DGluRIII mutant, we analyzed a series of lethal mutations in the region of DGluRIII (see Materials and Methods). We identified one complementation group containing two mutant alleles as the DGluRIII gene. Both alleles are embryonic lethal over a deficiency, as is the transheterozygote. Late-stage embryos appear grossly normal but are paralyzed and fail to hatch, which is consistent with a failure of neuromuscular transmission. The lethality of both alleles can be completely rescued by transgenic addition of a construct containing genomic DGluRIII.

Because we are particularly interested in the development of neuromuscular transmission during larval growth, we devised a method for generating DGluRIII hypomorphs that were viable throughout larval development. We generated flies that carried a DGluRIII cDNA construct under the control of the UAS promoter in the hopes of using mosaic expression to rescue the lethality. However, leaky expression of the UAS-DGluRIII cDNA in the absence of Gal4 was sufficient to rescue the lethality and provided us with a useful hypomorph. Staining of these "weakly" rescued flies reveals that DGluRIII levels are dramatically decreased (Fig. $4 a, b$ ). In contrast, full rescue of the same mutant with the genomic transgene restores highlevel expression of DGluRIII at the synapse (Fig. 4c). Despite the near absence of DGluRIII at the synapse, these weakly rescued flies appear to be healthy. They eclose as adults and have no obvious abnormalities with locomotion, flight, or fertility.

\section{DGluRIII is necessary for localization of DGluRIIA and DGluRIIB and for synaptic transmission}

To study interactions among the various glutamate receptor subunits, we examined synaptic expression of DGluRIIA and DGluRIIB in the DGluRIII loss of function mutants. We found that DGluRIIA and DGluRIIB are virtually undetectable at synapses expressing very low levels of DGluRIII (Fig. 4e,h). Full rescue of the DGluRIII mutant with the genomic transgene restores synaptic localization of both DGluRIIA and DGluRIIB (Fig. $4 f, i$ ). Hence, DGluRIII is essential for the synaptic localization of both DGluRIIA and DGluRIIB.

We next examined the physiological consequences of the near total absence of all known muscle glutamate receptor subunits. Electrophysiological recordings from wild-type muscles revealed $c-e, 6 \mu \mathrm{m} ; f-h, 12 \mu \mathrm{m}$.
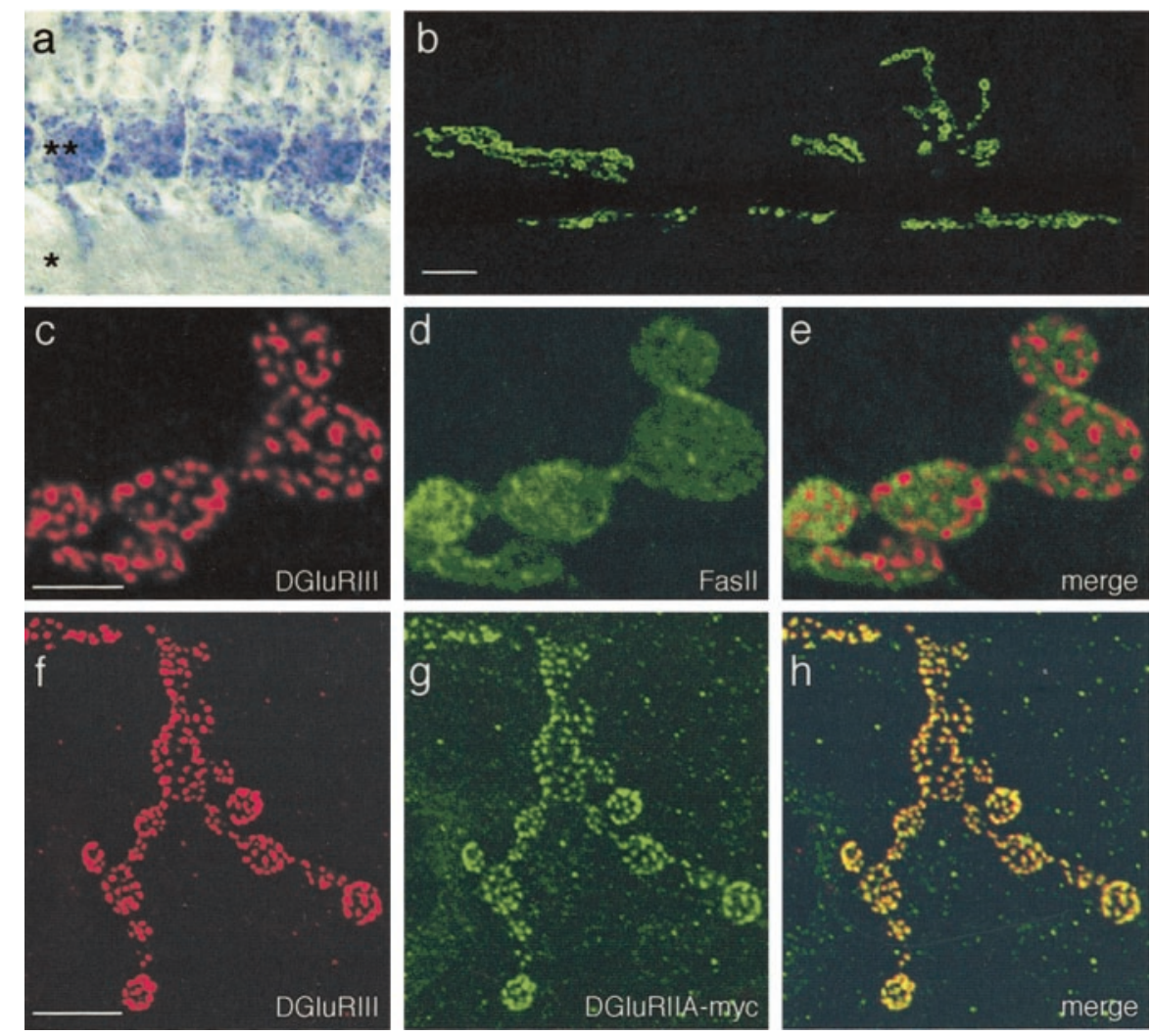

Figure 2. Expression pattern of DGluRIII. a, Embryonic in situ demonstrates the presence of DGluRIII transcript in the somatic musculature $\left(^{* *}\right)$ but not in the CNS $\left(^{*}\right) . b$, Confocal fluorescence microscopy of muscles 6 and 7 in a third-instar larvae reveals that DGluRIII is localized to the NMJ. $c-e$, High magnification view reveals that DGluRIII is localized to puncta, which are located in regions that fail to stain with FasIl. $f-h$, DGluRIII puncta colocalize with DGluRIIA-myc puncta, which are located opposite active zones in flies that express a myc-tagged DGluRIIA transgene (MHC-DGluRIIAmyc) (Petersen et al., 1997). Scale bars: $b, 15 \mu \mathrm{m}$;
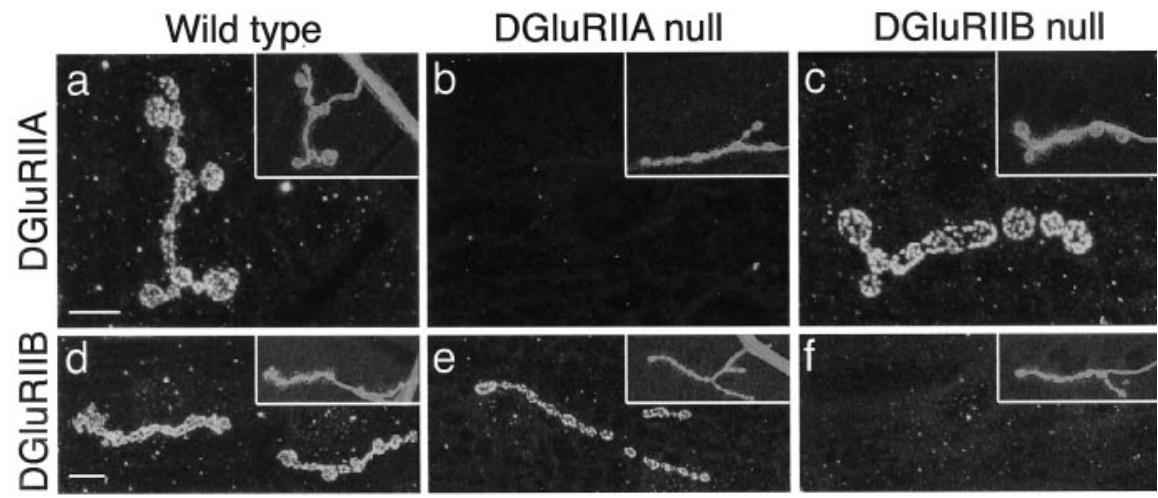

Figure 3. Antibodies for DGluRIIA and DGluRIIB are subunit specific. $a-c$, All visible anti-DGluRIIA staining disappears in DGluRIIA null mutants but is unaffected by the absence of DGluRIIB. $d-f$, All visible DGluRIIB staining disappears in DGluRIIB mutants but is unaffected by the absence of DGluRIIA. Anti-HRP (inset) shows the location of the nerve and synapse. Full genotypes are as follows: wild type, Canton S/W; DGluRIIA null, DGluRIIA ${ }^{A D 9} / D G l u R I I A \& B^{S P 22}$; DGluRIIB null, DGluRIIA\&B ${ }^{S P 22} / d f(2 L) C^{h 4}$; $P[g D G / u R I I A] /+$ (DGluRIIA and DGluRIIB double mutant rescued with a genomic DGluRIIA rescue construct). numerous miniature excitatory junctional potentials (mEJPs) (Fig. 5a). Each mEJP represents the postsynaptic depolarization in response to the spontaneous fusion of a single synaptic vesicle. In the DGluRIII weak rescue mutant, mEJPs are essentially undetectable; only rare and small events are observed (Fig. $5 b$ ). Very similar results were observed in 10 of 10 wild-type cells and 11 of 11 mutant cells. This indicates a dramatic loss in postsynaptic responsiveness to glutamate. Hence, DGluRIII is required for 

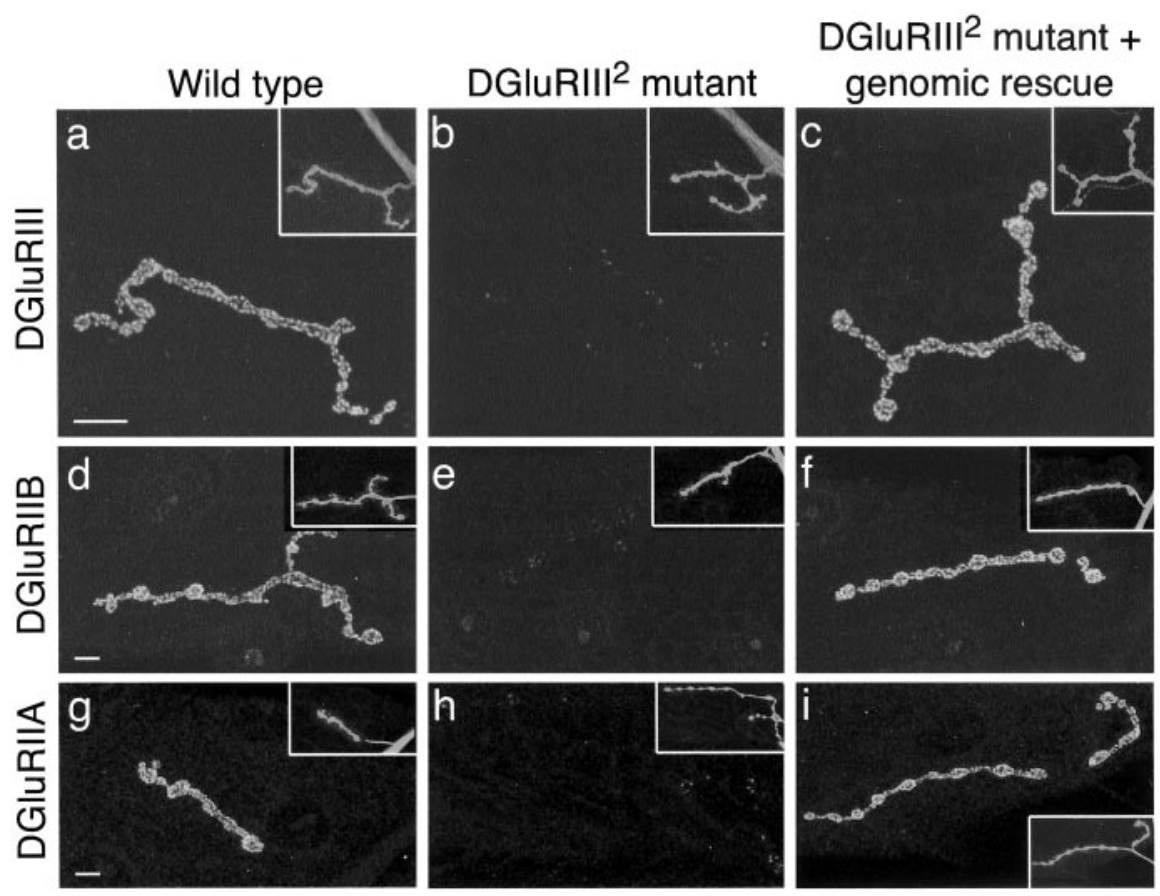

Figure 4. DGluRIII is required for synaptic localization of DGluRIIA and DGluRIIB. Confocal microscopy of muscle 4 using anti-glutamate receptor antibodies and anti-HRP (inset) is shown. $a-c$, DGluRIII levels are greatly reduced in strong DGluRIII hypomorphs (DGluRIII /df(2L)ast1;P[UAS-cGluRIII]/+) but are restored by the addition of genomic DGluRIII (DGluRIII /df(2L)ast 1; $P[g G l u R I I I] /+)$. Anti-HRP (inset) demonstrates normal synaptic structure in the hypomorphs. $d-i$, Both DGluRIIB $(d-f)$ and DGluRIIA $(g-i)$ are nearly undetectable in the near absence of DGluRIII but are present when DGluRIII levels are rescued. Scale bars: $a-c, 12 \mu \mathrm{m} ; d-i, 5 \mu \mathrm{m}$.

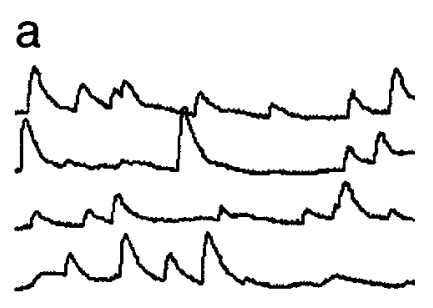

\section{Wild type}

Figure 5. DGluRIII is required for synaptic transmission. $a, b$, Representative traces of spontaneous transmitter release recorded from muscle 6 of wild-type $(a)$ and DGluRIII mutant $(b)$ third-instar larvae. There is almost no detectable response to the spontaneous fusion of single vesicles in the mutant. Full genotypes: $a$, wild type (Canton $\left.S / W^{-}\right) ; b$, DGluRIII mutant over deficiency rescued weakly with a cDNA transgene (DGluRIII'/df(2L)ast1; P[UAS-cDGluRIII]/+). Calibration: $2 \mathrm{mV}, 140 \mathrm{msec}$.

normal synaptic transmission at the Drosophila NMJ. The dearth of detectable mEJPs indicates that if other uncharacterized glutamate receptors are expressed at the NMJ, they are not sufficient to mediate robust synaptic transmission. However, we did observe robust evoked release in the mutant, indicating substantial homeostatic compensation, as has been observed in other glutamate receptor mutants (Petersen et al., 1997; DiAntonio et al., 1999; S.B.M. and A.D., unpublished observations).

\section{Competition between DGluRIIA and DGluRIIB}

Having demonstrated that DGluRIII is important for the localization of DGluRIIA and DGluRIIB, we assessed the role of DGluRIIA and DGluRIIB in the localization of DGluRIII. We found that the absence of either DGluRIIA or DGluRIIB has no effect on the synaptic localization of DGluRIII (Fig. $6 a-c$ ). Because DGlu-
RIIA and DGluRIIB are redundant for viability, we wondered whether the DGluRIIA and DGluRIIB double mutant would reveal a role for these subunits in the localization of DGluRIII. Although the double mutant is lethal, we previously generated a mosaic rescue system that allows us to analyze synapses in the absence of DGluRIIB and the near absence of DGluRIIA (DiAntonio et al., 1999). In muscles with extremely low levels of DGluRIIA and no DGluRIIB, we found that DGluRIII is barely detectable at the synapse (Fig. $6 d$ ). The occasional DGluRIII staining that is observed at these synapses corresponds with a punctum of weak DGluRIIA staining (data not shown). Together, our results indicate that DGluRIII and either DGluRIIA or DGluRIIB are required for the proper localization of the glutamate receptor to the NMJ. These data are consistent with a model in which these receptor subunits function together in a complex that includes DGluRIII in association with either DGluRIIA or DGluRIIB.

One prediction of the above model is that DGluRIIA and DGluRIIB would compete with each other for inclusion in the glutamate receptor complex. To test this prediction, we assessed the synaptic localization of all three subunits in wildtype larvae and larvae that overexpress either DGluRIIA or DGluRIIB. DGluRIII is unaffected by these manipulations; it is robustly expressed at the synapse in all three genotypes (Fig. $7 a-c$ ). However, the overexpression of either DGluRIIA or DGluRIIB has a profound effect on the synaptic localization of the other subunit (Fig. 7). Overexpression of DGluRIIB leads to a large decrease in the levels of DGluRIIA at the synapse (Fig. 7, compare $d$ and $f$ ). Overexpression of DGluRIIA gives the reciprocal phenotype; DGluRIIB is no longer detectable at the synapse in these larvae (Fig. 7, compare $g$ and $h$ ). The data shown in Figure 7 are for type Ib boutons on muscle 4; however, very similar results were observed at other muscles and at type Is boutons (data not shown). Together, these results indicate that DGluRIIA and DGluRIIB compete with each other, but not with DGluRIII, for access to the synapse.

What are DGluRIIA and DGluRIIB competing for? If DGluRIIA and DGluRIIB must assemble with DGluRIII to reach the synapse, then they may be competing for access to DGluRIII if its levels are limiting. To test this model, we co-overexpressed DGluRIIB and DGluRIII. We found that postsynaptic overexpression of DGluRIIB reduces the levels of DGluRIIA at the synapse to $27 \pm 2 \%$ of wild-type levels (MHC-DGluRIIB/ \pm ; $n=16$ ). However, when DGluRIII is simultaneously overexpressed with DGluRIIB, the levels of DGluRIIA at the synapse are significantly increased (52 $\pm 3 \%$ of wild-type levels, $M H C$-DGluRIIB/MHCDGluRIII; $n=16 ; p \ll 0.001)$. Hence, DGluRIIA and DGluRIIB compete with each other for access to DGluRIII.

\section{Afferents regulate the differential localization of DGluRIIA and DGluRIIB}

Having identified competitive interactions between DGluRIIA and DGluRIIB in mutant larvae, we assessed whether these recep- 
Wild type

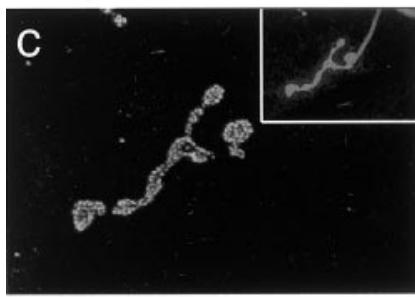

DGluRIIB null

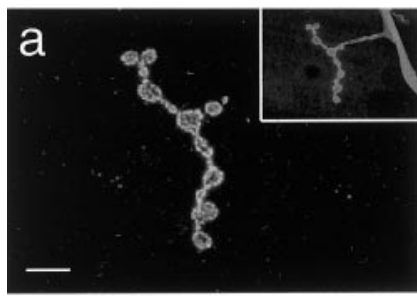

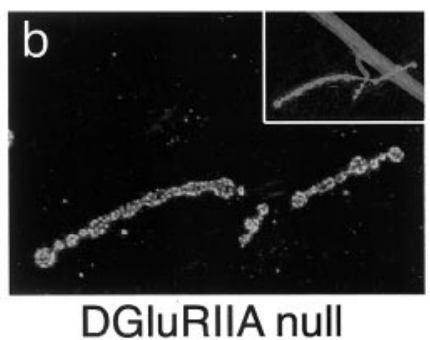

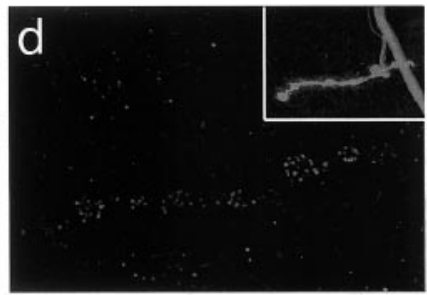

DGluRIIA hypomorph DGluRIIB null
Figure 6. Either DGluRIIA or DGluRIIB is required for synaptic localization of DGluRIII. Confocal microscopy was performed on muscle 4 with anti-DGluRIII and anti-HRP (inset). DGluRIII staining appears normal in larvae that are genetically null for either DGIURIIA or DGIURIIB but is barely visible in muscles that lack DGluRIIB and have only low levels of DGluRIIA. Full genotypes: $a$, wild type, Canton $S / w^{-} ; b$, DGluRIIA null, DGluRIIA $A^{A D 9} / D$ GluRIIA\&B ${ }^{S P 22} ; c$, DGluRIIB null, DGluRIIA\&B ${ }^{S P 22} / d f(2 L) c^{h 4}$; P[gDGluRIIA]/+; d, DGluRIIA hypomorph, DGluRIIB null, DGluRIIA\&B ${ }^{\text {SP22 }} / d f(2 L) c^{\text {h4; }}$ H94Gal4/P[UAS-DGluRIIAmyc]. Scale bar, $10 \mu \mathrm{m}$.

tor subunits are differentially used at wild-type synapses. Drosophila muscle cells are multi-innervated by different classes of motoneurons that have distinct morphological and physiological properties (Atwood et al., 1993; Jia et al., 1993; Kurdyak et al., 1994; Lnenicka and Keshishian, 2000; Hoang and Chiba, 2001). Each muscle receives innervation from a type Ib motoneuron, whereas most also receive innervation from type Is and/or type II motoneurons. We double stained wild-type larvae for both DGluRIIA and DGluRIIB. We observed robust staining of DGluRIIA and DGluRIIB at type Ib synapses (Fig. 8, asterisk). However, synapses made by other motoneurons onto the same postsynaptic cells show a differential utilization of DGluRIIA and DGluRIIB. The most striking difference was observed on muscle 8, where DGluRIIB is strongly expressed at the NMJ of both inputs, whereas DGluRIIA is only highly expressed at the type Ib input [Fig. $8 a, b,{ }^{*}$, \# (synapses from the two motoneurons)]. The identities of the innervating motoneurons onto muscle 8 are not well defined; however, we found a similar difference between the type $\mathrm{Ib}$ and type Is innervations of the well characterized lateral muscles $6,7,13$, and 12 (Fig. 8c-f). DGluRIIB is highly expressed at both classes of synapses, whereas DGluRIIA is less strongly expressed at the Is than the Ib synapse. For each cell, we calculated the intensity of staining for each receptor at the type Is and Ib synapses. For DGluRIIB, the ratio of Is to Ib is $1.21 \pm 0.05(n=$ $14)$, whereas for DGluRIIA, the ratio of Is to Ib is $0.66 \pm 0.05$ ( $n=$ $14)$. Hence, the distribution of glutamate receptor subunits opposite each motoneuron is significantly different $(p \ll 0.001)$. We found that DGluRIII, the essential subunit, is highly expressed at all of these synapses (Fig. $8 f$ ). To investigate whether this differential localization of DGluRIIA and DGluRIIB changes the physiology of the synapse, we assessed the kinetics of the excitatory junctional currents from the type Ib and type Is neurons. As predicted from the single-channel properties of these channels (DiAntonio et al., 1999), the synaptic currents from the type Is neuron are significantly faster than from the type Ib neuron (width at half-maximum; mean $\pm \mathrm{SE}$; Is, $7.48 \pm 0.23 \mathrm{msec}$; Ib, $8.34 \pm 0.18 \mathrm{msec} ; n=7 ; p=0.012$ ). These anatomical and physiological results indicate that afferents converging on a single postsynaptic target may regulate the subunit composition of postsynaptic receptors.

Having observed differences in the localization of DGluRIIA and DGluRIIB at different synapses, we assessed their localization at individual receptor puncta at synapses in which they are both highly expressed. For this, we analyzed individual confocal slices at high resolution. We observed very little colocalization of DGluRIIA and DGluRIIB. Instead, DGluRIIB often forms a ring, whereas DGluRIIA is a patch that fills the hole in the DGluRIIB ring (Fig. $8 g$ ). Although no single confocal slice shows this ring and spot appearance for every receptor puncta, analysis of the entire stack indicates that this is the most common arrangement of receptors. This demonstrates that these subunits are differentially localized even within a single receptor patch, suggesting that they rarely, if ever, coassemble into the same glutamate receptor complex.

Finally, we investigated glutamate receptor localization to the less well characterized type II synapses. Type II NMJs are primarily peptidergic, although they stain for glutamate, which could be released as a cotransmitter (Johansen et al., 1989; Jia et al., 1993). We observed faint staining of DGluRIII and DGluRIIA at type II NMJs of multiple muscles but did not detect DGluRIIB (Fig. $9 a-c)$. Hence, this class of synapse appears to be glutamatergic as well as peptidergic. The differential utilization of DGluRIIA and DGluRIIB allowed us to test whether these two subunits compete for DGluRIII or for access to synaptic anchoring sites. Overexpression of DGluRIIA, which is normally present at type II synapses, leads to more robust expression of DGluRIII at type II synapses (Fig. 9d). However, overexpression of DGluRIIB, which is not present at type II synapses, drove DGluRIII away from type II synapses (Fig. 9e). It is not displaced by DGluRIIB, because even overexpressed DGluRIIB is still not detectable at these synapses (Fig. 9f). Because DGluRIIB expression redistributes DGluRIII away from type II synapses, although it is not present at these synapses, this supports the model that DGluRIIB is competing with DGluRIIA for DGluRIII and not for access to binding sites at these synapses.

\section{Discussion}

In this study, we investigated the localization of glutamate receptor subunits at the Drosophila NMJ. We previously described two subunits, DGluRIIA and DGluRIIB, that have distinct physiological properties but are redundant for viability (Petersen et al., 1997; DiAntonio et al., 1999). We now identify a novel glutamate receptor subunit, DGluRIII, and demonstrate that it is an essential gene required for the synaptic localization of DGluRIIA and DGluRIIB and for synaptic transmission. We found that either DGluRIIA or DGluRIIB, but not both, is required for the synaptic localization of DGluRIII. These two redundant subunits compete with each other for access to DGluRIII. Together, these results suggest the existence of a receptor complex that must include DGluRIII and either DGluRIIA or DGluRIIB. The presence of two interchangeable, yet physiologically distinct, subunits provides the cell with opportunities for synapse-specific regulation of synaptic function. DGluRIIA and DGluRIIB are differentially localized to the postsynaptic membrane opposite the terminals of distinct motoneurons that converge on the same postsynaptic cell. Hence, afferents may regulate postsynaptic receptor subunit choice. This study defines molecules and mechanisms that con- 


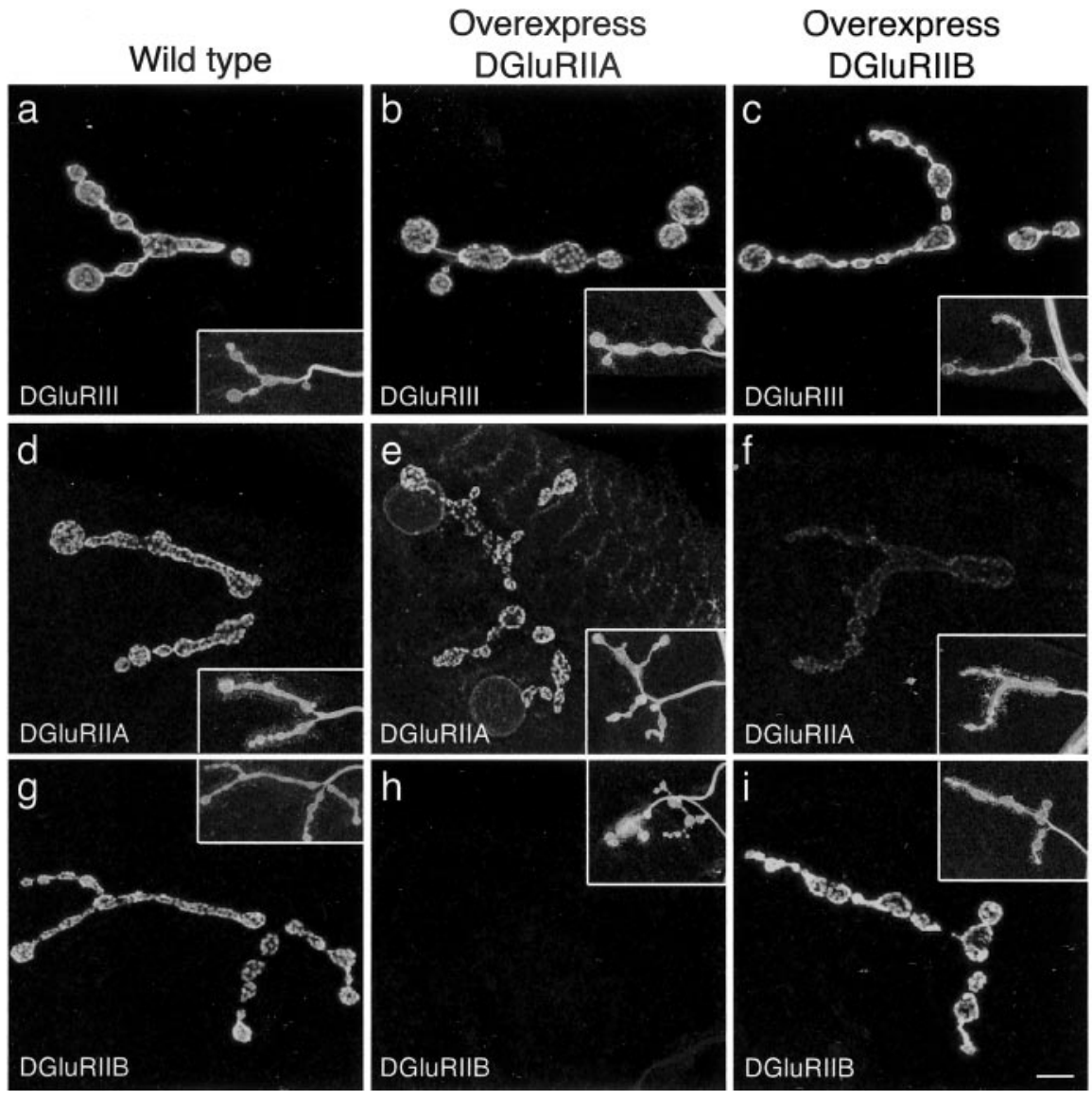

Figure 7. Competition between DGluRIIA and DGluRIIB. $a-i$, Confocal microscopy was performed on muscle 4 with antiDGluRIII ( $a-c)$, anti-DGluRIIA ( $d-f)$, anti-DGluRIIB $(g-i)$, and anti-HRP (inset) from larvae that are genetically wild type (Canton S) $(a, d, g)$, overexpress DGluRIIA (MHC-DGluRIIA) $(b, e, h)$, or overexpress DGluRIIB (MHC-DGluRIIB) $(c, f, i)$. Overexpression of either DGluRIIA or DGluRIIB reduces the synaptic localization of the other but does not affect the localization of DGluRIII. Scale bar, $8 \mu \mathrm{m}$. apses, which are the classic glutamatergic input, as well as by type II synapses, which are thought to be peptidergic. Although the presynaptic terminals of type II motoneurons are filled with dense-core vesicles, they also stain strongly for glutamate (Johansen et al., 1989; Jia et al., 1993). It is not known whether these synapses mediate ionotropic or metabotropic glutamatergic transmission or whether they even release glutamate. We observed DGluRIII at type II synapses as well as type I synapses, albeit at much lower levels. Hence, it is likely that type II synapses are able to mediate ionotropic glutamatergic synaptic transmission, potentially releasing glutamate as a cotransmitter with neuropeptides.

\section{Glutamate receptor interactions}

Mammalian glutamate receptors are composed of multiple subunits. Recent experiments indicate that the functional receptor is a tetramer and that assembly of the receptor occurs through a "dimerization of dimers" (Ayalon and Stern-Bach, 2001). Insertion of the receptor into the synaptic membrane is regulated by the precise subunit composition (Barry and Ziff, 2002). Our results are consistent with a similar model for Drosophila glutamate receptors. We found that the presence of DGluRIII is required for the localization of DGluRIIA and DGluRIIB to the NMJ, and that either DGluRIIA or DGluRIIB is required for the synaptic localization of DGluRIII. Moretrol the localization of glutamate receptors at the Drosophila neuromuscular junction and highlights the role of the nerve in shaping the synaptic properties of its postsynaptic partner.

DGluRIII is a novel glutamate receptor at the Drosophila NMJ To define the molecular composition of the glutamate receptor complex at the Drosophila NMJ, we identified and characterized a novel muscle-expressed receptor that we named DGluRIII (previously known as CG4226). Based on sequence homology, DGluRIII is most closely related to the previously described Drosophila muscle glutamate receptors DGluRIIA and DGluRIIB. In situ hybridization reveals that somatic muscles of embryos and thirdinstar larvae express the DGluRIII transcript. Immunocytochemistry with an antibody recognizing DGluRIII demonstrates that it is expressed at the neuromuscular junction. We found that DGluRIII is located in discrete puncta at type I synapses. These puncta are located in the gaps in FasII staining, which were previously shown to represent active zones (Sone et al., 2000; Wan et al., 2000) and colocalize with myc-tagged DGluRIIA receptors that are present opposite EM-identified active zones (Petersen et al., 1997). Because transgenic myc-DGluRIIA is present opposite every active zone (Petersen et al., 1997) and because DGluRIII colocalizes with every myc-DGluRIIA punctum, it is likely that DGluRIII is present opposite every active zone. Hence, the antibody against DGluRIII may provide a useful reagent to assess the number of active zones using light microscopy.

Many Drosophila muscles are innervated by both type I syn- over, DGluRIIA and DGluRIIB compete with each other, but not with DGluRIII, for synaptic localization. These observations are consistent with a model in which these molecules are part of a complex, and that DGluRIII is a required component that must interact with either DGluRIIA or DGluRIIB. Although acting as genetic nulls, the molecular lesions in the DGluRIII mutants are not defined, and thus it remains a formal possibility that a molecularly defined null could indicate another function for DGluRIII. The inability of individual subunits to localize to the synapse suggests that receptor interactions are necessary for either assembly and trafficking of receptors to the synapse or stabilization of receptors at the synapse.

We previously observed a competition between DGluRIIA and DGluRIIB at the level of synaptic function. Overexpression of DGluRIIA leads to a dose-dependent increase in quantal size, whereas overexpression of DGluRIIB leads to a dose-dependent decrease in quantal size (Petersen et al., 1997; DiAntonio et al., 1999). Hence, the relative levels of these two subunits are an important determinant of postsynaptic sensitivity to glutamate. Sigrist et al. (2002) also observed a competition between these two subunits; they found that overexpression of DGluRIIA modulates synaptic morphology and that this phenotype can be suppressed by the co-overexpression of DGluRIIB. Our demonstration that DGluRIIA and DGluRIIB compete with each other for access to DGluRIII and subsequent localization to the synapse provides a cell biological explanation for these previous findings.

What role might each subunit play in the synaptic localization 

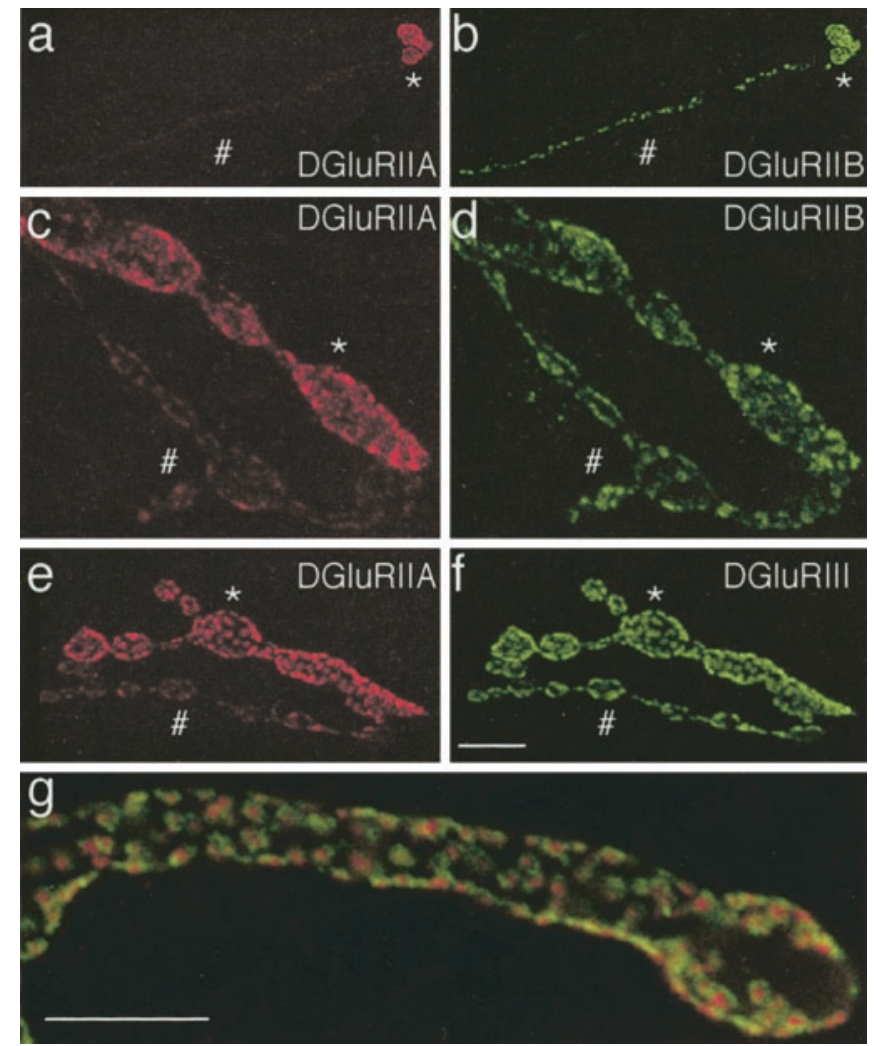

Figure 8. DGluRIIA and DGluRIIB are differentially localized within a single postsynaptic cell. Confocal microscopy was used to assess the localization of glutamate receptor subunits onto single postsynaptic muscles that are innervated by two motoneurons $\left({ }^{*}, \#\right) . a, b, 0$ n muscle 8 DGluRIIB is abundant opposite both inputs $(b)$, whereas DGluRIIA is only abundant at the type Ib input $(a) . c, d$, Similar results are seen at muscle 13 where DGluRIIB is abundant at both types $\mathrm{lb}$ $\left({ }^{*}\right)$ and Is (\#) NMJ (d), whereas DGluRIIA is expressed at higher levels at the type lb $\left(^{*}\right)$ input (c). $e, f$, Similar results are found at muscle 13 when comparing the expression of DGluRIII ( $f$ ) with DGluRIIA (e). $g$, High-resolution images of a type lb synapse show that DGluRIIA (red) and DGluRIIB (green) are not extensively colocalized within receptor puncta. Scale bars: $a, b, 10 \mu \mathrm{m}$ $c, d, 8 \mu \mathrm{m} ; e, f, 9 \mu \mathrm{m} ; g, 6 \mu \mathrm{m}$.

of the glutamate receptor complex? In many systems, proteins containing the PDZ domain play important anchoring and structural roles in synapse organization (Hung and Sheng, 2002). It is interesting that DGluRIII alone among the Drosophila muscle receptors contains a predicted $\mathrm{PDZ}$-binding domain at the cytoplasmic $C$ terminus. The sequence at the terminus is -EARV, which is a class II PDZ-binding domain (-X- $\Phi-\mathrm{X}-\Phi$, where $\Phi$ is any hydrophobic amino acid) (Hung and Sheng, 2002). Although it remains possible that internal PDZ-binding domains may be present in DGluRIIA or DGluRIIB, it is an intriguing possibility that DGluRIII contains essential localization signals. However, because DGluRIII requires either DGluRIIA or DGluRIIB for proper localization, this PDZ-binding domain is not sufficient to target this receptor to the synapse. In fact, DGluRIIA and DGluRIIB must themselves contain important localizationstabilization signals. These two subunits are expressed at different levels at distinct NMJs on a single postsynaptic cell. In addition, they localize to different domains within a single postsynaptic receptor patch. Hence, DGluRIIA and DGluRIIB must contain targeting information that is not present in DGluRIII. Defining the role of each subunit will require the identification of their binding partners and of the synaptic anchoring proteins used at each type of neuromuscular junction.

\section{Afferents regulate the composition of postsynaptic} glutamate receptors

When presynaptic inputs that use different neurotransmitters, such as GABA or glutamate, converge on a single postsynaptic target, the postsynaptic receptors that cluster at each terminal match the presynaptic transmitter phenotype (Craig et al., 1994; Craig and Boudin, 2001). Hence, the afferent regulates the postsynaptic receptor choice. There is much less evidence that afferents regulate the subunit composition of a particular receptor type. A few studies using pharmacological and physiological analysis at vertebrate glutamatergic synapses suggest that such regulation does occur. Toth and McBain (1998, 2000) studied mossy fiber and CA3 commissural afferent inputs onto single hippocampal interneurons. They found that philanthotoxin-433, which blocks GluR2-lacking AMPA receptors, blocked mossy fiber but not CA3 synaptic transmission. Hence, there is afferentspecific utilization of AMPA receptor subunits. However, what is not clear in this system is the spatial distribution of synapses. If the afferents innervate distinct compartments of the interneuron, then the subunit choice could be a consequence of differential sorting of receptors by the postsynaptic cell and not be induced by the afferents. A similar phenomenon has recently been observed for NMDA receptor subunits. Inputs from the left and right sides of the brain converging on a single hippocampal neuron show differential sensitivity to an antagonist of channels containing the NR2B (NMDA receptor 2B) subunit (Kawakami et al., 2003). In the present study, we took advantage of the singlecell resolution achievable in the insect nervous system to investigate this issue with direct anatomical observation.

In the Drosophila neuromuscular system, each muscle is unique and identifiable. Most muscles are multi-innervated by identified motoneurons that make synapses with different morphological and physiological properties. Each muscle coexpresses the essential glutamate receptor subunit DGluRIII as well as the DGluRIIA and DGluRIIB subunits. However, the levels of these three subunits differ at distinct synapses within a single muscle. If we take muscle 13 as an example, it is innervated by three motoneurons, each of which makes a different class of synapse (types Ib, Is, and II). In muscle 13, DGluRIII is present at high levels at the type Ib and Is synapses and is present at the type II synapse. DGluRIIB is present at high levels at the type Ib and Is synapses but is not detected at the type II synapse. Finally, DGluRIIA is present at high levels at the type Ib synapse, at lower levels at the type Is synapse, and is present at the type II synapse. Hence, each motoneuron contacts a postsynaptic density with a different complement of glutamate receptor subunits. Because DGluRIIA and DGluRIIB have different single-channel properties that affect the synaptic response (DiAntonio et al., 1999), this differential localization of receptor subunits may regulate the synaptic function and plasticity of the NMJ of each afferent. We previously found that lower levels of DGluRIIA, relative to DGluRIIB, lead to a smaller quantal size (Petersen et al., 1997) and report here that DGluRIIA levels are relatively lower at type Is boutons. However, the quantal size is slightly higher at type Is boutons than at type Ib boutons (Karunanithi et al., 2002). This apparent paradox may be resolved by the observation that at type Is boutons, synaptic vesicles are larger and, hence, contain more glutamate (Karunanithi et al., 2002).

How does the identity of the presynaptic motoneuron regulate the postsynaptic subunit composition? One hypothesis from the vertebrate work is that the postsynaptic cell targets subunits to distinct compartments, and that afferents then innervate different compartments. This is unlikely at the Drosophila NMJ. The 


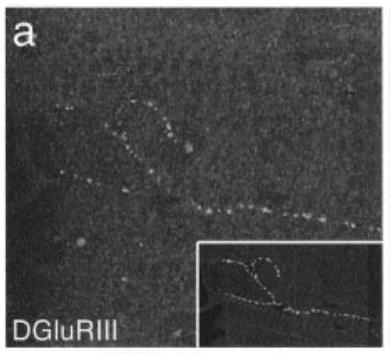

Wild type

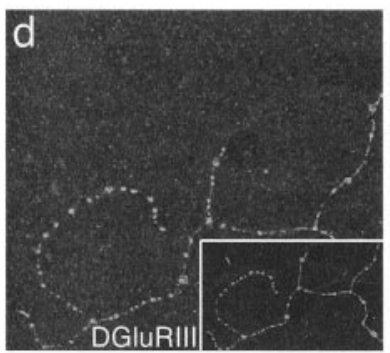

Overexpress

DGluRIIA

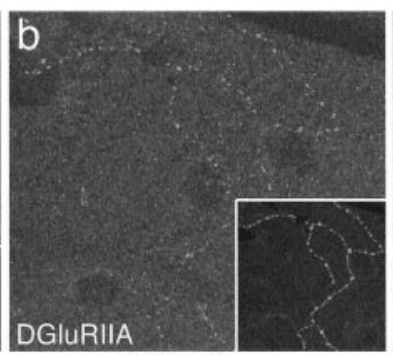

Wild type

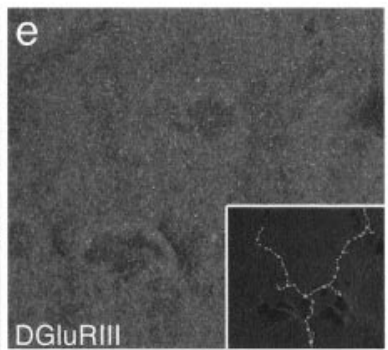

Overexpress DGluRIIB

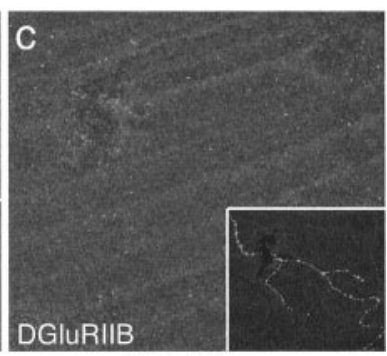

Wild type

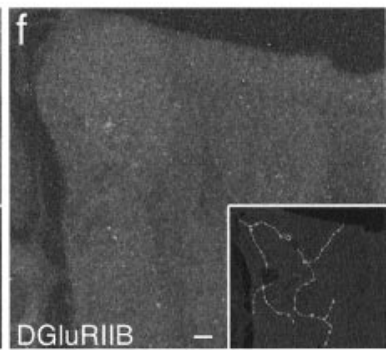

Overexpress DGluRIIB

Figure 9. Glutamate receptor subunits are expressed at type II synapses. Confocal microscopy was used to assess the localization of glutamate receptor subunits at type II synapses of muscle 31 in segment A1. $a-c$, In wild-type larvae, DGluRIII (a) and DGluRIIA $(b)$ are detectable, but DGluRIIB is not ( $c$ ). $d$, When DGluRIIA is overexpressed (genotype MHC-DGluRIIA), DGluRIII is still observed at type II NMJs. However, when DGluRIIB is overexpressed $(e, f ;$ genotype MHC-DGluRIIB), DGluRIII is no longer detectable at these synapses (e). Even when overexpressed, DGluRIIB is not detected at type II NMJs ( $f$ ). In all panels, anti-HRP staining reveals the shape of the nerve (insets). Scale bar, $30 \mu \mathrm{m}$.

postsynaptic muscle has a very simple shape, and the distinct synapses can be adjacent to each other, so each neuron is probably not innervating a distinct compartment. A second model is that there is a temporal, as opposed to spatial, compartment, with the timing of receptor expression and motoneuron innervation determining the subunits that predominate opposite each nerve. This is unlikely because all three receptors are expressed and new boutons are added throughout development (Schuster et al., 1996; Petersen et al., 1997; Zito et al., 1999). We favor an alternate model in which the neuron recruits particular subunits to its synapse. This could occur via distinct molecular cues or because of differences in activity among the different classes of motoneurons. The possibility that activity is the key factor is consistent with the recent finding of experience-dependent changes in the expression of DGluRIIA (Sigrist et al., 2003). The genetic and anatomical tools available in the Drosophila neuromuscular system should allow us to test these models in the future.

\section{References}

Atwood HL, Govind CK, Wu CF (1993) Differential ultrastructure of synaptic terminals on ventral longitudinal abdominal muscles in Drosophila larvae. J Neurobiol 24:1008-1024.

Ayalon G, Stern-Bach Y (2001) Functional assembly of AMPA and kainate receptors is mediated by several discrete protein-protein interactions. Neuron 31:103-113.

Barry MF, ZiffEB (2002) Receptor trafficking and the plasticity of excitatory synapses. Curr Opin Neurobiol 12:279-286.

Brand AH, Perrimon N (1993) Targeted gene expression as a means of altering cell fates and generating dominant phenotypes. Development 118:401-415.

Craig AM, Boudin H (2001) Molecular heterogeneity of central synapses: afferent and target regulation. Nat Neurosci 4:569-578.

Craig AM, Blackstone CD, Huganir RL, Banker G (1994) Selective clustering of glutamate and gamma-aminobutyric acid receptors opposite ter- minals releasing the corresponding neurotransmitters. Proc Natl Acad Sci USA 91:12373-12377.

Davis GW, DiAntonio A, Petersen SA, Goodman CS (1998) Postsynaptic PKA controls quantal size and reveals a retrograde signal that regulates presynaptic transmitter release in Drosophila. Neuron 20:305-315.

DiAntonio A, Petersen SA, Heckmann M, Goodman CS (1999) Glutamate receptor expression regulates quantal size and quantal content at the Drosophila neuromuscular junction. J Neurosci 19:3023-3032.

Dingledine R, Borges K, Bowie D, Traynelis SF (1999) The glutamate receptor ion channels. Pharmacol Rev 51:7-61.

Hoang B, Chiba A (2001) Single-cell analysis of Drosophila larval neuromuscular synapses. Dev Biol 229:55-70.

Hollmann M, Heinemann S (1994) Cloned glutamate receptors. Annu Rev Neurosci 17:31-108.

Hung AY, Sheng M (2002) PDZ domains: structural modules for protein complex assembly. J Biol Chem 277:5699-5702.

Jia XX, Gorczyca M, Budnik V (1993) Ultrastructure of neuromuscular junctions in Drosophila: comparison of wild type and mutants with increased excitability. J Neurobiol [Erratum (1994) 25:893-895] 24:1025-1044.

Johansen J, Halpern ME, Johansen KM, Keshishian H (1989) Stereotypic morphology of glutamatergic synapses on identified muscle cells of Drosophila larvae. J Neurosci 9:710-725.

Jonas P, Racca C, Sakmann B, Seeburg PH, Monyer H (1994) Differences in Ca2+ permeability of AMPA-type glutamate receptor channels in neocortical neurons caused by differential GluR-B subunit expression. Neuron 12:1281-1289.

Karunanithi S, Marin L, Wong K, Atwood HL (2002) Quantal size and variation determined by vesicle size in normal and mutant Drosophila glutamatergic synapses. J Neurosci 22:10267-10276.

Kawakami R, Shinohara Y, Kato Y, Sugiyama H, Shigemoto R, Ito I (2003) Asymmetrical allocation of NMDA receptor epsilon2 subunits in hippocampal circuitry. Science 300:990-994.

Kurdyak P, Atwood HL, Stewart BA, Wu CF (1994) Differential physiology and morphology of motor axons to ventral longitudinal muscles in larval Drosophila. J Comp Neurol 350:463-472.

Littleton JT, Ganetzky B (2000) Ion channels and synaptic organization: analysis of the Drosophila genome. Neuron 26:35-43.

Lnenicka GA, Keshishian H (2000) Identified motor terminals in Drosophila larvae show distinct differences in morphology and physiology. J Neurobiol 43:186-197.

Malinow R, Malenka RC (2002) AMPA receptor trafficking and synaptic plasticity. Annu Rev Neurosci 25:103-126.

McGee AW, Bredt DS (2003) Assembly and plasticity of the glutamatergic postsynaptic specialization. Curr Opin Neurobiol 13:111-118.

Petersen SA, Fetter RD, Noordermeer JN, Goodman CS, DiAntonio A (1997) Genetic analysis of glutamate receptors in Drosophila reveals a retrograde signal regulating presynaptic transmitter release. Neuron 19:1237-1248.

Schuster CM, Ultsch A, Schloss P, Cox JA, Schmitt B, Betz H (1991) Molecular cloning of an invertebrate glutamate receptor subunit expressed in Drosophila muscle. Science 254:112-114.

Schuster CM, Davis GW, Fetter RD, Goodman CS (1996) Genetic dissection of structural and functional components of synaptic plasticity. I. Fasciclin II controls synaptic stabilization and growth. Neuron 17:641-654.

Sheng M, Pak DT (1999) Glutamate receptor anchoring proteins and the molecular organization of excitatory synapses. Ann NY Acad Sci 868:483-493.

Sigrist SJ, Thiel PR, Reiff DF, Schuster CM (2002) The postsynaptic gluta- 
mate receptor subunit DGluR-IIA mediates long-term plasticity in Drosophila. J Neurosci 22:7362-7372.

Sigrist SJ, Reiff DF, Thiel PR, Steinert JR, Schuster CM (2003) Experiencedependent strengthening of Drosophila neuromuscular junctions. J Neurosci 23:6546-6556.

Sone M, Suzuki E, Hoshino M, Hou D, Kuromi H, Fukata M, Kuroda S, Kaibuchi K, Nabeshima Y, Hama C (2000) Synaptic development is controlled in the periactive zones of Drosophila synapses. Development 127:4157-4168.

Stewart BA, Atwood HL, Renger JJ, Wang J, Wu CF (1994) Improved stability of Drosophila larval neuromuscular preparations in haemolymphlike physiological solutions. J Comp Physiol [A] 175:179-191.

Tautz D, Pfeifle C (1989) A non-radioactive in situ hybridization method for the localization of specific RNAs in Drosophila embryos reveals translational control of the segmentation gene hunchback. Chromosoma 98:81-85.

Toth K, McBain CJ (1998) Afferent-specific innervation of two distinct AMPA receptor subtypes on single hippocampal interneurons. Nat Neurosci 1:572-578.

Toth K, McBain CJ (2000) Target-specific expression of pre- and postsynaptic mechanisms. J Physiol (Lond) 525:41-51.

Ultsch A, Schuster CM, Laube B, Schloss P, Schmitt B, Betz H (1992) Glu- tamate receptors of Drosophila melanogaster: cloning of a kainateselective subunit expressed in the central nervous system. Proc Natl Acad Sci USA 89:10484-10488.

Ultsch A, Schuster CM, Laube B, Betz H, Schmitt B (1993) Glutamate receptors of Drosophila melanogaster. Primary structure of a putative NMDA receptor protein expressed in the head of the adult fly. FEBS Lett 324:171-177.

Vèolkner M, Lenz-Bèohme B, Betz H, Schmitt B (2000) Novel CNS glutamate receptor subunit genes of Drosophila melanogaster. J Neurochem 75:1791-1799.

Wan HI, DiAntonio A, Fetter RD, Bergstrom K, Strauss R, Goodman CS (2000) Highwire regulates synaptic growth in Drosophila. Neuron 26:313-329.

Washburn MS, Numberger M, Zhang S, Dingledine R (1997) Differential dependence on GluR2 expression of three characteristic features of AMPA receptors. J Neurosci 17:9393-9406.

Westbrook GL (1994) Glutamate receptor update. Curr Opin Neurobiol 4:337-346.

Zito K, Parnas D, Fetter RD, Isacoff EY, Goodman CS (1999) Watching a synapse grow: noninvasive confocal imaging of synaptic growth in Drosophila. Neuron 22:719-729. 